\title{
A PROOF OF AN IDENTITY FOR MULTIPLICATIVE FUNCTIONS
}

\author{
K. KRISHNA
}

Introduction. An arithmetic function $f$ is said to be multiplicative if $f(m n)=$ $f(m) f(n)$, whenever $(m, n)=1$ and $f(1)=1$. The Dirichlet convolution of two arithmetic functions $f$ and $g$, denoted by $f \cdot g$, is defined by $f \cdot g(n)=$ $\sum_{d \mid n} f(d) g(n / d)$. Let $w(n)$ denote the product of the distinct prime factors of $n$, with $w(1)=1$. R. Vaidyanathaswamy [3] proved the following identical equation for any multiplicative arithmetic function $f$ :

$$
f(m n)=\sum_{\substack{a|m \\ b| n}} f(m / a) f(n / b) f^{-1}(a b) C(a, b),
$$

where $m$ and $n$ are arbitrary positive integers, $f^{-1}$ is the Dirichlet inverse of $f$ defined by

$$
\sum_{d \mid n} f(d) f^{-1}(n / d)=E_{o}(n)=\left\{\begin{array}{lll}
1 & \text { if } & n=1 \\
0 & \text { if } & n>1
\end{array}\right.
$$

and $C(a, b)$ is a multiplicative function of two variables defined by

$$
C(a, b)=\left\{\begin{array}{cl}
(-1)^{k} & \text { if } w(a)=w(b)=k \\
0 & \text { otherwise. }
\end{array}\right.
$$

The $K$-product of any two arithmetic functions $f$ and $g$ is the arithmetic function $f \times g$ defined by

$$
f \times g(n)=\sum_{d \mid n} f(d) g(n / d) K((d, n / d)),
$$

where $K(n)$ is a fixed arithmetic function satisfying $K(1)=1$ and, for arbitrary positive integers $a, b, c$,

$$
K((a, b)) K((a b, c))=K((a, b c)) K((b, c)) .
$$

It has been shown [1] that (2) assures the associativity of the $K$-product and, together with the condition $K(1)=1$, it implies that $K(n)$ is multiplicative.

M. V. Subba Rao and A. A. Gioia [2] gave a generalization of the identity

Received by the editors March 29, 1978 and, in revised form, September 10, 1978. 
(1), which holds in the case of the $K$-product. The generalized identity is

$$
f(m n)=\sum_{\substack{a|m \\ b| n}} f(m / a) f(n / b) f^{-1}(a b) K((m n / a b, a b)) K((m / a, n / b)) C(a, b) .
$$

Their proof of (3) is based on the observation that the right side of (3) actually defines a multiplicative function of both the variables $m$ and $n$ so that one need only evaluate it when $m$ and $n$ are prime powers. The object of this note is to point out a new proof of (3) which is a straightforward generalization of Vaidyanathaswamy's proof of (1).

LEMMA 1. Let $f$ be any multiplicative function and $f^{-1}$ be its inverse with respect to the $K$-product operation. Then, for arbitrary positive integers $m_{1}, m_{2}$ and $n$, the sum

$$
\sum f\left(m_{1} d\right) f^{-1}\left(m_{2} n / d\right) K\left(\left(m_{1} d, m_{2} n / d\right)\right),
$$

extended over all the divisors $d$ of $n$, vanishes unless every prime factor of $n$ divides $m_{1} m_{2}$.

Proof. Let $n=n_{1} n_{2}$, where all the prime factors of $n_{1}$ divide $m_{1} m_{2}$, and $n_{2}$ is relatively prime to $m_{1} m_{2}$. Then it is clear that $\left(n_{1}, n_{2}\right)=1$, and therefore any factor $d$ of $n$ can be expressed uniquely in the form $d_{1} d_{2}$, where $d_{1}$ is a divisor of $n_{1}$ and $d_{2}$ is a divisor of $n_{2}$.

Hence we have

$$
\begin{aligned}
\sum f\left(m_{1} d\right) f^{-1}\left(m_{2} n / d\right) & K\left(\left(m_{1} d, m_{2} n / d\right)\right) \\
= & \sum f\left(m_{1} d_{1} d_{2}\right) f^{-1}\left(m_{2} n_{1} / d_{1} \cdot n_{2} / d_{2}\right) K\left(\left(m_{1} d_{1} d_{2}, m_{2} n_{1} n_{2} / d_{1} d_{2}\right)\right) \\
= & \left\{\sum f\left(m_{1} d_{1}\right) f^{-1}\left(m_{2} n_{1} / d_{1}\right) K\left(\left(m_{1} d_{1}, m_{2} n_{1} / d_{1}\right)\right)\right\} \\
& \times\left\{\sum f\left(d_{2}\right) f^{-1}\left(n_{2} / d_{2}\right) K\left(\left(d_{2}, n_{2} / d_{2}\right)\right)\right\},
\end{aligned}
$$

where we have used the multiplicativity of $f$ and $f^{-1}$ together with the relation (see Lemma in section 3 of [2]):

(4) $K((a b, c d))=K((a, c)) K((b, d)) \quad$ if $\quad(a, b)=1,(a, d)=1 \quad$ and $\quad(b, c)=1$.

Now the summation in the second curly bracket above vanishes unless $n_{2}=1$, which proves the result.

COROllary. Calling a factor $n_{1}$ of $n$ a block factor if $\left(n_{1}, n / n_{1}\right)=1$, we have

$$
\sum f(n / d) f^{-1}(d) K((n / d, d))=0,
$$

where the summation extends over all the divisors $d$ of a block factor $n_{1}(\neq 1)$ of $n$. 
LEMMA 2. Let $w(n)=\nu$. Then

$$
\sum_{\substack{d \mid n \\ w(d)=w(n)}} f(n / d) f^{-1}(d) K((n / d, d))=(-1)^{\nu} f(n) .
$$

Proof. Let $n_{i 1}, n_{i 2}, \ldots, n_{i k}\left(k=\left(\begin{array}{l}\nu \\ i\end{array}\right)\right)$ denote the distinct block factors of $n$ which contain exactly $i$ of the prime factors. Consider the sum

$$
\begin{aligned}
A= & \sum_{n} f(n / d) f^{-1}(d) K((n / d, d))-\sum_{k=1}^{\nu}\left\{\sum_{n_{\nu-1 k}} f(n / d) f^{-1}(d) K((n / d, d))\right\} \\
& +\sum_{k=1}^{\nu(\nu-1) / 2}\left\{\sum_{n_{\nu-2 k}} f(n / d) f^{-1}(d) K((n / d, d))\right\}-\cdots \\
& +(-1)^{\nu-1} \sum_{k=1}^{\nu}\left\{\sum_{n_{1 k}} f(n / d) f^{-1}(d) K((n / d, d))\right\},
\end{aligned}
$$

where the $n_{i j}$ below $\sum$ indicates that the sum is extended over all the divisors $d$ of $n_{i j}$. We evaluate the expression $A$ in two ways. First, we observe that every partial sum in $A$, except the first, vanishes by the corollary to Lemma 1 . Hence we have,

$$
A=\sum_{n} f(n / d) f^{-1}(d) K((n / d, d))=0, \quad(n>1) .
$$

On the other hand consider a particular divisor $d$ of $n$, containing $i$ distinct prime factors. The coefficient of $f(n / d) f^{-1}(d) K((n / d, d))$ in $A$ is

$$
1-\left(\begin{array}{c}
\nu-i \\
1
\end{array}\right)+\left(\begin{array}{c}
\nu-i \\
2
\end{array}\right)-\cdots=\left\{\begin{array}{lll}
0 & \text { if } & 0<i<\nu \\
1 & \text { if } & i=\nu
\end{array}\right.
$$

If $d=1$, the coefficient of $f(n / 1) f^{-1}(1) K((n / 1,1))$ is

$$
1-\left(\begin{array}{l}
\nu \\
1
\end{array}\right)+\left(\begin{array}{l}
\nu \\
2
\end{array}\right)-\cdots+(-1)^{\nu-1}\left(\begin{array}{c}
\nu \\
\nu-1
\end{array}\right)=(-1)^{\nu-1}
$$

Therefore we have

$$
A=\sum_{\substack{d \mid n \\ w(d)=w(n)}} f(n / d) f^{-1}(d) K((n / d, d))+(-1)^{\nu-1} f(n) .
$$

But we have already observed that $A=0$. Hence we obtain the required identity.

LemMa 3. Let $w(m)=w(n)=\nu$. Then

$$
\sum_{b \mid n} f(m n / b) f^{-1}(b) K((m n / b, b))=(-1)^{\nu} \sum_{\substack{a \mid m \\ w(a)=w(m)}} f(m / a) f^{-1}(n a) K((m / a, n a))
$$


Proof. The proof is analogous to the proof of Theorem 3 of [3]. We shall just outline the proof here.

Let $m=m_{i k} m_{i k}^{\prime}$ and $n=n_{i k} n_{i k}^{\prime}$, where $m_{i k}$ and $n_{i k}\left(k=1,2, \ldots,\left(\begin{array}{c}\nu \\ i\end{array}\right)\right)$ are the block factors of $m$ and $n$ respectively, which contain the same $i$ prime factors. Hence $\left(m_{i k}, m_{i k}^{\prime}\right)=1,\left(n_{i k}, n_{i k}^{\prime}\right)=1$, and $m_{i k}^{\prime}$ and $n_{i k}^{\prime}$ are the block factors of $m$ and $n$ respectively, containing the same $(\nu-i)$ prime factors.

Consider the expression

$$
\begin{aligned}
B= & \sum f(m n / b) f^{-1}(b) K((m n / b, b)) \\
& +\sum_{k=1}^{\nu}\left\{\sum \sum f\left(m_{1 k} / a \cdot m_{1 k}^{\prime} n_{1 k}^{\prime} / b\right) f^{-1}\left(n_{1 k} a b\right) K\left(\left(m_{1 k} / a \cdot m_{1 k}^{\prime} n_{1 k}^{\prime} / b, n_{1 k} a b\right)\right)\right\} \\
& -\sum_{k=1}^{\nu(\nu-1) / 2}\left\{\sum \sum f\left(m_{2 k} / a \cdot m_{2 k}^{\prime} n_{2 k}^{\prime} / b\right) f^{-1}\left(n_{2 k} a b\right) K\left(\left(m_{2 k} / a \cdot m_{2 k}^{\prime} n_{2 k}^{\prime} / b, n_{2 k} a b\right)\right)\right\} \\
& +\cdots+(-1)^{\nu-1} \sum f(m / a) f^{-1}(n b) K((m / a, n b)) .
\end{aligned}
$$

Here the first term of $B$ is a summation over all divisors $b$ of $n$. Every succeeding term contains three summations; the two inner summations relate respectively to all divisors $b$ of $m_{i k}^{\prime} n_{i k}^{\prime}$ and to all such divisors $a$ of $m_{i k}^{\prime}$ which contain all its distinct prime factors; the outer summation relates to all possible resolutions of $m$ and $n$ into corresponding block factors containing $i$ and $(\nu-i)$ primes. The signs of the $(\nu+1)$ terms in $B$ alternate from the second term onwards. In the last term $i=\nu$, and so the outer summation as well as the summation relating to $b$, has disappeared, leaving only the summation over all factors $a$ of $m$ containing all its $\nu$ prime factors.

The proof is now complete after the evaluation of the expression $B$ in two ways, as we have done in the previous lemma.

Corollary 1. Let $w(m)=w(n)=\nu$ and $\left(m_{1}, m\right)=1$, and hence $\left(m_{1}, n\right)=1$. Put $m^{\prime}=m_{1} m$. Then, multiplying both sides of (5) by $f\left(m_{1}\right) K\left(\left(m_{1}, 1\right)\right)$, we get, on using (4) and the multiplicativity of $f$

$$
\begin{aligned}
\sum_{b \mid n} f\left(m^{\prime} n / b\right) f^{-1}(b) K\left(\left(m^{\prime} n / b, b\right)\right) & =(-1)^{\nu} \sum_{\substack{a \mid m^{\prime} \\
w(a)=w(n)}} f\left(m^{\prime} / a\right) f^{-1}(n a) K\left(\left(m^{\prime} / a, n a\right)\right) .
\end{aligned}
$$

COROllary 2. Let $m$ and $n$ be any two positive integers, with $w(n)=\nu$. Then

$$
\sum_{b \mid n} f(m n / b) f^{-1}(b) K((m n / b, b))=(-1)^{\nu} \sum_{\substack{a \mid m \\ w(a)=w(n)}} f(m / a) f^{-1}(n a) K((m / a, n a)) .
$$

Proof. If $w(n) \mid w(m)$, then this reduces to Corollary 1 above. If $w(n) \nmid$ $w(m)$, the left side is zero by Lemma 1 , while the right side is an empty sum. 
We can now prove the generalized identical equation for $K$-products:

THEOREM. If $f$ is multiplicative, then for any two positive integers $m$ and $n$,

$$
f(m n)=\sum_{\substack{a|m \\ b| n}} f(m / a) f(n / b) f^{-1}(a b) K((m n / a b, a b)) K((m / a, n / b)) C(a, b) .
$$

Proof. From Corollary 2, with $n_{1}$ in the place of $n$, we have

(6) $\sum_{b / n_{1}} f\left(m n_{1} / b\right) f^{-1}(b) K\left(\left(m n_{1} / b, b\right)\right)$

$$
=(-1)^{\nu} \sum_{\substack{a \mid m \\ w(a)=w\left(n_{1}\right)}} f(m / a) f^{-1}\left(n_{1} a\right) K\left(\left(m / a, n_{1} a\right)\right),
$$

where $\nu=w\left(n_{1}\right)$.

We multiply both sides of (6) by $f\left(n_{2}\right) K\left(\left(n_{2}, m n_{1}\right)\right)$, and sum over all values of $n_{1}$ and $n_{2}$ with $n_{1} n_{2}=n$. The summation is carried out in two stages; namely, we first keep $n_{1} / b$ fixed, and sum over all values of $n_{2}$ and $b$ such that $n_{2} b=n b / n_{1}$. On the left side, by using relation (2), we get

$$
\begin{aligned}
\sum_{n_{1} n_{2}=n} \sum_{b \mid n_{1}} f\left(m n_{1} / b\right) f^{-1}(b) K\left(\left(m n_{1} / b, b\right)\right) f\left(n_{2}\right) K\left(\left(n_{2}, m n_{1}\right)\right) \\
=\sum_{n_{1} n_{2}=n} \sum_{b \mid n_{1}} f\left(m n_{1} / b\right) f^{-1}(b) f\left(n_{2}\right) K\left(\left(m n_{1} / b, n_{2} b\right)\right) K\left(\left(n_{2}, b\right)\right), \\
=\sum f\left(m n_{1} / b\right) K\left(\left(m n_{1} / b, n_{2} b\right)\right) \sum_{n_{2} b=n b / n_{1}} f\left(n_{2}\right) f^{-1}(b) K\left(\left(n_{2}, b\right)\right) .
\end{aligned}
$$

The second summation here vanishes, by Lemma 1 , unless $n b / n_{1}=1$ (equivalently $n_{2} b=1$ ), that is, unless $n_{1}=n b$, in which case it is 1 . Therefore the left side of $(6)$ reduces to $f(m n) K((m n, 1))=f(m n)$.

The right side of (6), after multiplying by $f\left(n_{2}\right) K\left(\left(n_{2}, m n_{1}\right)\right)$, is

$$
\sum_{n_{1} n_{2}=n} \sum_{\substack{a \mid m \\ w(a)=w\left(n_{1}\right)}}(-1)^{v} f(m / a) f^{-1}\left(n_{1} a\right) K\left(\left(m / a, n_{1} a\right)\right) f\left(n_{2}\right) K\left(\left(n_{2}, m n_{1}\right)\right),
$$

which is equal to

$$
\sum \sum(-1)^{\nu} f(m / a) f(n / b) f^{-1}(a b) K((m / a, a b)) K((n / b, m b)),
$$

where we sum over all the divisors $b$ of $n$ and all the divisors $a$ of $m$ with $w(a)=w(b)$.

This, by the definition of $C(a, b)$ and by the relation (2), is clearly equal to

$$
\sum_{\substack{a|m \\ b| n}} f(m / a) f(n / b) f^{-1}(a b) K((m n / a b, a b)) K((m / a, n / b)) C(a, b),
$$

and the proof of the theorem is complete. 
Acknowledgement. I wish to thank Dr. R. Sitaramachandra Rao, Andhra University, Waltair, India, for having brought my attention to this problem.

A good part of this work was done when the author was in the University of Mysore, Manasa Gangotri, India.

\section{REFERENCES}

1. A. A. Gioia, The K-product of Arithmetic Functions, Canad. J. Math. 17 (1965), 970-976.

2. M. V. Subba Rao and A. A. Gioia, Identities for multiplicative functions, Canad. Math. Bull. 10 (1967), 65-73.

3. R. Vaidyanathaswamy, The identical equations of the multiplicative function, Bull. Amer. Math. Soc. 36 (1930), 762-772.

DEPARTMENT OF MATHEMATICS

UNIVERSITY OF PITTSBURGH

Pittsburgh, PenN, 15260 